\section{FATORES ASSOCIADOS À APTIDÃO CARDIORRESPIRATÓRIA DE ESCOLARES}

\author{
FACTORS ASSOCIATED WITH CARDIORESPIRATORY FITNESS IN SCHOOL STUDENTS
}

FACTORES ASOCIADOS A LA APTITUD CARDIORRESPIRATORIA DE LOS ESCOLARES

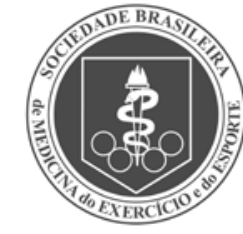

Artigo Original

Original ARTICLE

Artículo Original
Diogo Henrique Constantino Coledam (Educador Físico)

Philippe Fanelli Ferraiol ${ }^{2}$

(Educador Físico)

Júlio Wilson dos-Santos ${ }^{3}$

(Educador Físico)

Arli Ramos de Oliveira ${ }^{2}$

(Educador Físico)

1. Instituto Federal de São Paulo (IFSP), São Paulo, SP, Brasil.

2. Universidade Estadual de Londrina, UEM/UEL (UEL), Londrina, PR, Brasil. 3. Universidade Estadual Paulista (UNESP), Instituto de Biociências, Bauru, SP. Brasil.

\section{Correspondência:}

Instituto Federal de Educação,

Ciência e Tecnologia de São Paulo

(IFSP) Campus Boituva.

Av. Zélia de Lima Rosa, 100. Portal

dos Pássaros, Boituva, SP, Brasil.

18550-000 diogohcc@yahoo.com.br

\section{RESUMO}

Introdução: Há alta prevalência de jovens que não atendem ao critério de saúde para aptidão cardiorrespiratória, o que os expõe a riscos relacionados à saúde metabólica, cardiovascular e mental. Dessa forma, é relevante investigar quais aspectos estão associados à aptidão cardiorrespiratória de jovens. Objetivo: Analisar os fatores associados à aptidão cardiorrespiratória em escolares. Métodos: Estudo transversal que envolveu 736 escolares (50,1\% do sexo feminino) com idade entre 10 a 18 anos. Foram analisadas a condição socioeconômica, escolaridade paterna, número de irmãos, percepção de atividade física, atividade física moderada a vigorosa, participação nas aulas de educação física, prática esportiva, local para prática, transporte ativo e comportamento sedentário. Foram coletadas medidas de estatura, massa corporal e realizado um teste de aptidão cardiorrespiratória. Para estimar a razão de prevalência (RP) e os intervalos de confiança de 95\% foi utilizada a regressão de Poisson. Resultados: Para escolares com idade inferior a 14 anos, as variáveis que apresentaram associação com a aptidão cardiorrespiratória foram o sexo feminino ( $R P=0,79,0,68-0,91)$, sobrepeso ( $R P=0,73,0,61-0,89$ ), percepção de atividade física igual às pessoas de mesma idade $(R P=1,26,1,03-1,53)$, atividade física semanal de 3-6h ( $R P=1,21,1,05-1,38)$, participação nas aulas de educação física ( $R P=1,35,1,03-1,76)$ e comportamento sedentário $\geq 2 h(R P=0,82,0,73-0,94)$. Com relação aos escolares com idade igual ou superior a 14 anos, o sexo feminino ( $R P=0,35,0,19-0,63)$, o sobrepeso ( $R P=0,25,0,09-0,66)$, praticar esportes algumas vezes ( $R P=1,96$, 1,00-3,81) e frequentemente ( $\mathrm{RP}=2,09,1,01-4,31)$ apresentaram associação com a aptidão cardiorrespiratória. Conclusão: Programas de intervenção com o objetivo de aumentar o atendimento ao critério de saúde para aptidão cardiorrespiratória de escolares devem promover a prática de atividade física moderada a vigorosa, prática esportiva, participação nas aulas de educação física e redução do sobrepeso.

Descritores: aptidão física; atividade motora; educação física e treinamento; sobrepeso; adolescente.

\section{ABSTRACT}

Introduction: There is a high prevalence of youth that not meet the health criterion for cardiorespiratory fitness, which exposes them to risks related to metabolic, cardiovascular and mental health. Thus, it is relevant to investigate which aspects are associated with cardiorespiratory fitness in youth. Objective: To analyze the factors associated with cardiorespiratory fitness in school students. Methods: Cross-sectional study involving 736 students (50.1\% female) with age between 10 and 18 years. The following has been analyzed: the socioeconomic condition, paternal education, number of siblings, physical activity perception, moderate to vigorous physical activity, participation in physical education classes, sport practice, place to practice, active transport and sedentary behavior. Height and body mass were measured and a cardiorespiratory fitness test was performed. Prevalence ratio (PR) and $95 \%$ confidence intervals were estimated using the Poisson regression. Results: For students aged less than 14 years, variables that presented association with cardiorespiratory fitness were female sex $(P R=0.79,0.68-0.91)$, overweight $(P R=0.73,0.61-0.89)$, physical activity perception equal to persons of the same age ( $P R=1.26,1.03-1.53)$, weekly physical activity 3-6h $(P R=1.21,1.05-1.38)$, participation in physical education classes $(P R=1.35,1.03-1.76)$, and sedentary behavior $\geq 2 \mathrm{~h}$ $(R P=0.82,0.73-0.94)$. With respect to students aged 14 years or more, female sex $(P R=0.35,0.19-0.63)$, overweight $(P R=0.25,0.09-0.66)$, practicing sports a few times $(P R=1.96,1.00-3.81)$ and frequently $(P R=2.09,1.01-4.31)$ presented association with cardiorespiratory fitness. Conclusion: Intervention programs aiming at increasing health criterion requirement for cardiorespiratory fitness of students should promote moderate to vigorous physical activity, sport practice, participation in physical education classes and overweight reduction.

Keywords: physical fitness; motor activity; physical education and training; overweigh; adolescent.

\section{RESUMEN}

Introducción: Existe una alta prevalencia de jóvenes que no cumplen los criterios de salud para aptitud cardiorrespiratoria, que los expone a riesgos relacionados con la salud metabólica, cardiovascular y mental. De este modo, es importante investigar qué aspectos están asociados con la aptitud cardiorrespiratoria de los jóvenes. Objetivo: Analizar los factores relacionados con la aptitud cardiorrespiratoria en escolares. Métodos: Estudio transversal que implica 736 escolares (50,1\% mujeres) de 10 a 18 años de edad. Fueron analizados el nivel socioeconómico, escolaridad paterna, número de hermanos, percepción de la actividad física, actividad física moderada a 
vigorosa, participación en las clases de educación física, práctica deportiva, lugar para práctica, transporte activo y el comportamiento sedentario. Fueron tomadas las medidas de talla, masa corporal y llevado a cabo una prueba de aptitud cardiorrespiratoria. Para estimar la razón de prevalencia (RP) y los intervalos de confianza de 95\% se utilizó la regresión de Poisson. Resultados: En los escolares menores de 14 años, las variables que se asociaron con la aptitud cardiorrespiratoria fueron el sexo femenino $(R P=0,79,0,68$ a 0,91), exceso de peso $(R P=0,73,0,61-0,89)$, percepción de actividad física igual a las personas de la misma edad ( $R P=1,26,1,03-1.53$ ), actividad física semanal 3-6h $(R P=1,21,1,05-1,38)$, participación en las clases de educación física ( $R P=1,35,1,03-1,76)$ y el comportamiento sedentario $\geq 2 h(R P=0,82,0,73-0,94)$. Con respecto a los escolares mayores de 14 años, el sexo femenino $(R P=0,35,0,19-0,63)$, exceso de peso ( $R P=0,25,0,09-0,66)$, práctica de deportes algunas veces $(R P=1,96,1,00-3,81)$ y frecuentemente $(R P=2,09,1,01-4,31)$ se asociaron con la aptitud cardiorrespiratoria. Conclusión: Los programas de intervención con el propósito de aumentar el cumplimiento de los criterios de salud para la aptitud cardiorrespiratoria en escolares deben promover la práctica de actividad física moderada a vigorosa, práctica deportiva, participación en las clases de educación física y la reducción del exceso de peso.

Descriptores: aptitud fisica; actividad motora; educación y entrenamiento físico; sobrepeso; adolescente.

\section{INTRODUÇÃO}

A aptidão cardiorrespiratória é um aspecto positivamente associado à saúde metabólica, cardiovascular ${ }^{1}$ e mental ${ }^{2}$ de adolescentes. Além disso, a aptidão cardiorrespiratória é melhor preditor do risco cardiovascular quando comparado à atividade física ${ }^{3,4}$, embora a associação possa ser mediada pelo percentual de gordura ${ }^{4}$.

No Brasil, a prevalência de atendimento ao critério de saúde para aptidão cardiorrespiratória em jovens já foi descrita e varia de 20 a $68.5 \% \%^{5-10}$. Devido a alta prevalência de aptidão cardiorrespiratória inadequada, estudos buscaram identificar os fatores associados em crianças e adolescentes brasileiros ${ }^{6-11}$. Três estudos analisaram apenas os fatores sociodemográficos em adolescentes de 14 a 19 anos $6,7,9$ e os outros analisaram fatores sociodemográficos e comportamentais em jovens de 6 a 18 anos $^{8,10,11}$.

Estudos realizados no Brasil demonstraram que a aptidão cardiorrespiratória está associada ao sexo e idade ${ }^{6}$, condição socioeconômica, escolaridade dos pais, trabalho remunerado, transporte ativo, atividade física ${ }^{10,11}$, área de domicílio ${ }^{7,9}$, comportamento sedentário ${ }^{8,10}$ e sobrepeso $^{12}$. Apesar disso, esses estudos possuem a limitação de não terem analisado em um mesmo modelo os fatores descritos além de outros que podem estar associados à aptidão cardiorrespiratória de escolares, tais como participação nas aulas de Educação Física, prática esportiva, disponibilidade de locais para prática de atividade física, percepção de atividade física e número de irmãos.

Do ponto de vista epidemiológico, identificar os fatores associados à aptidão cardiorrespiratória de escolares poderá fornecer informações relevantes para a implementação de políticas públicas junto às escolas e a comunidade com objetivo de aumentar a proporção de escolares que atendem aos critérios de saúde. Além disso, poderá nortear futuros estudos com objetivo de identificar outros fatores associados ou estudos de caráter experimental, com objetivo de analisar programas de intervenção visando a melhora da aptidão cardiorrespiratória de escolares. Dessa forma, o objetivo desse estudo foi analisar os fatores associados à aptidão cardiorrespiratória de escolares.

\section{MÉTODOS}

Estudo transversal realizado na rede estadual de ensino da cidade de Londrina-PR, Brasil no ano de 2012. Nesse ano havia 55.475 alunos matriculados na rede estadual de ensino do sexto ano do ensino fundamental ao terceiro ano do ensino médio. Os escolares estavam distribuídos nas regiões da cidade na seguinte proporção: Norte (20,5\%),
Sul (18\%), Leste (17\%), Oeste (15,5\%) e Centro (29\%). O método para a seleção amostral foi o probabilístico, utilizando conglomerados e estratificado por sexo e região da cidade (norte, sul, leste, oeste e centro), realizado em dois estágios. Primeiramente foi sorteado aleatoriamente uma escola de cada região da cidade e de cada escola foi avaliada a quantidade proporcional ao número de alunos da região, utilizando as salas de aulas completas.

O cálculo amostral foi realizado utilizando como parâmetros: população de 55,475 estudantes matriculados no ensino médio na cidade de Londrina-PR no ano de 2012, prevalência de atendimento ao critério de aptidão cardiorrespiratória utilizando os pontos de corte propostos pela FITNESSGRAM de $38 \%{ }^{5}$, erro amostral de 5\%, intervalo de confiança de 95\%, efeito de delineamento de dois devido a amostragem complexa utilizada e acréscimo de 15\% para possíveis perdas. Sendo assim o número necessário de participantes no estudo foi de 720 escolares. No presente estudo foram avaliados 965 escolares. No entanto, a amostra final foi constituída por 736 participantes, uma vez que foram analisados apenas os dados dos indivíduos que preencheram todas as questões e realizaram todos os procedimentos do estudo. Os critérios de inclusão foram: estar matriculado na rede estadual de ensino entre o $6^{\circ}$ ano do Ensino Fundamental e $3^{\circ}$ ano do Ensino Médio, ter entre 10 e 18 anos de idade e não possuir nenhuma limitação física/ortopédica que impedisse a realização dos procedimentos do estudo.

O estudo foi aprovado pelo Comitê de Ética em Pesquisa envolvendo Seres Humanos da Universidade Estadual de Londrina-PR sob protocolo n 312/2011, de acordo com a resolução 196/96 do Conselho Nacional de Saúde. Todos os responsáveis pelos escolares que aceitaram participar do estudo assinaram um Termo de Consentimento Livre e Esclarecido, constando todos os procedimentos que seriam realizados e formas de contatos para esclarecimento de possíveis dúvidas.

Todos os procedimentos foram realizados na escola onde os alunos se encontravam matriculados. Em sala de aula os alunos responderam a um questionário, com objetivo de estimar a atividade física semanal ${ }^{13}$, transporte ativo ${ }^{13}$, percepção de atividade física ${ }^{13}$, prática esportiva ${ }^{13}$, escolaridade paterna ${ }^{14}$, comportamento sedentário, número de irmãos, local para a prática de atividade física e participação nas aulas de Educação Física ${ }^{15}$. As questões utilizadas são descritas no Quadro 1. No mesmo dia, os participantes realizaram medidas antropométricas e na quadra da escola o teste de aptidão cardiorrespiratória.

A condição socioeconômica foi estimada por meio dos Critérios de Classificação Econômica do Brasil ${ }^{14}$, que estabelece classificações para o nível socioeconômico de acordo com a estimativa de renda média 
Quadro 1. Descrição do questionário utilizado no estudo.

\begin{tabular}{|c|c|c|}
\hline Variável & Questão & Opções de resposta \\
\hline \multirow{2}{*}{ Atividade física semanal ${ }^{16}$} & $\begin{array}{l}\text { O esporte/programa de exercícios físicos que você mais frequentemente } \\
\text { pratica apresenta intensidade:* }\end{array}$ & ( ) Baixa, ( ) Moderada, ( ) Elevada \\
\hline & $\begin{array}{l}\text { Durante quantas horas/semana você pratica este esporte/programa de } \\
\text { exercícios físicos? }{ }^{+}\end{array}$ & ( ) $<1$ hora, ( ) 1-2 horas, ( ) 2-3 horas, ( ) 3-4 horas, ( ) $>4$ horas \\
\hline Transporte ativo ${ }^{16}$ & $\begin{array}{l}\text { Durante quanto tempo por dia você caminha e/ou anda de bicicleta para } \\
\text { ir ao trabalho, à escola e às compras? }\end{array}$ & $\begin{array}{l}\text { ( ) }<5 \text { minutos ( ) 5-15 minutos } \\
\text { ( ) } 15-30 \text { minutos ( ) 30-45 minutos } \\
\text { ( ) } 45 \text { minutos }\end{array}$ \\
\hline Percepção de atividade física ${ }^{16}$ & $\begin{array}{l}\text { Em comparação com outras pessoas de mesma idade, você acredita que } \\
\text { as atividades que realiza durante seu tempo livre são fisicamente: }\end{array}$ & $\begin{array}{l}\text { ( ) Muito elevadas, ( ) Elevadas } \\
\text { ( ) Iguais ( ) Baixas ( ) Muito baixas }\end{array}$ \\
\hline Prática esportiva ${ }^{16}$ & Nas atividades de lazer e de ocupação do tempo livre você pratica esportes: & $\begin{array}{l}\text { ( ) Nunca ( ) Raramente ( ) Algumas vezes } \\
\text { ( ) Frequentemente ( ) Sempre }\end{array}$ \\
\hline Escolaridade paterna ${ }^{17}$ & Coloque o grau de instrução de seu pai: & 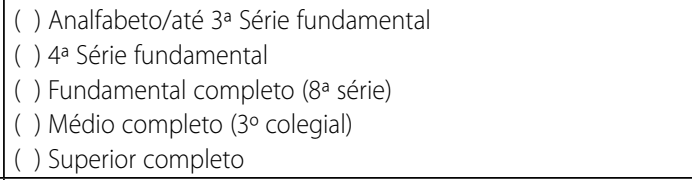 \\
\hline Comportamento sedentário & $\begin{array}{l}\text { Quantas horas, em média, você assiste TV, joga videogame ou usa o } \\
\text { computador? }\end{array}$ & $\begin{array}{l}\text { ( ) }<1 \text { hora por dia ( ) } 1 \text { hora por dia } \\
\text { ( ) } 2 \text { horas por dia ( ) } 3 \text { horas por dia } \\
\text { ( ) } 4 \text { horas por dia ( ) } 5 \text { ou mais horas por dia }\end{array}$ \\
\hline Número de irmãos & Quantos irmãos (ãs) você possui? & Aberta \\
\hline $\begin{array}{l}\text { Local para a prática de ativi- } \\
\text { dade física }\end{array}$ & $\begin{array}{l}\text { No seu bairro existe algum lugar adequado (praça, parque, quadra, campo } \\
\text { ou clube) para você jogar, brincar ou praticar algum esporte? }\end{array}$ & $\begin{array}{l}\text { ( ) Não } \\
\text { ( ) Sim, apenas um } \\
\text { ( ) Sim, mais que um }\end{array}$ \\
\hline $\begin{array}{l}\text { Participação nas aulas de Edu- } \\
\text { cação Física }{ }^{15}\end{array}$ & Neste semestre, você participou das aulas de Educação Física? & $\begin{array}{l}\text { ( ) Sim } \\
\text { ( ) Não }\end{array}$ \\
\hline
\end{tabular}

'Responderam a questão apenas os jovens que declararam praticar atividade física. ${ }^{\dagger} \mathrm{A}$ mesma questão foi respondida novamente caso o jovem praticasse outra atividade física.

familiar: A1 (R\$ 11,480), A2 (R\$ 8,295), B1 (R\$ 4,754), B2 (R\$ 2,656), C1 $(R \$ 1,459), C 2(R \$ 962), D(R \$ 680)$ e $E(R \$ 415)$. Para análise as categorias foram agrupadas em alta (A1 e A2), média (B1, B2) e baixa (C1, C2, D e E).

O estado nutricional foi estimado por meio do Índice de Massa Corporal, calculado por meio da equação massa corporal (kg)/estatura(m)². A estatura foi medida por meio de uma fita métrica fixada na parede (Sanny, São Paulo, Brasil), com precisão de $1 \mathrm{~mm}$ e a massa corporal total por meio de uma balança eletrônica com precisão de $100 \mathrm{~g} \mathrm{e}$ capacidade de 150 kg (Plenna, modelo MEA-03140, São Paulo, Brasil), de acordo com procedimentos já descritos ${ }^{18}$. Os escolares foram classificados com sobrepeso de acordo com International Obesity Task Force ${ }^{19}$.

A aptidão cardiorrespiratória foi avaliada por meio do teste PACER (Progressive Aerobic Cardiovascular Endurance Run) ${ }^{18}$. O critério de saúde utilizado foi o proposto pela FITNESSGRAM ${ }^{19}$, e os escolares foram classificados em atende (zona saudável de aptidão) e não atende (algum risco), de acordo com a faixa etária e sexo.

A reprodutibilidade do questionário utilizado no presente estudo foi testada em 70 escolares, no intervalo de sete dias. Para as variáveis categóricas, todas as questões apresentaram reprodutibilidade aceitável, com a concordância das questões testada por meio do índice Kappa variando de 65 a 100\% entre os dois momentos. Para a questão com variável numérica discreta (número de irmãos) a reprodutibilidade foi alta, com coeficiente de correlação intraclasse $=0,964$ (0,926-0,983).

A estatística descritiva foi realizada utilizando a frequência relativa. A reprodutibilidade das variáveis categóricas foi analisada utilizando o índice Kappa e para a variável numérica utilizou-se o coeficiente de correlação intraclasse de um fator aleatório. Para analisar a associação bivariada entre as variáveis independentes e o atendimento do critério para aptidão cardiorrespiratória foi utilizado o teste de Qui-quadrado. As variáveis que apresentaram $p \leq 0,20$ foram incluídas na análise multivariada, realizada por meio da regressão de Poisson com ajuste robusto de variância para estimar as razões de prevalências e os respectivos intervalos de confiança de 95\%, sendo que a significância adotada foi de $5 \%$.

\section{RESULTADOS}

As características descritivas dos participantes são apresentadas na Tabela 1.

A associação entre aptidão cardiorrespiratória e as variáveis independentes estudadas são descritas na Tabela 2 para os escolares com idade inferior a 14 anos. Na análise ajustada as variáveis sexo feminino $(R P=0,79)$, sobrepeso $(R P=0,73)$, percepção de atividade física igual as pessoas de mesma idade ( $R P=1,26)$, realizar 3 a 6 h atividade física moderada a vigorosa semanal $(\mathrm{RP}=1,21)$, participação nas aulas de Educação Física $(\mathrm{RP}=1,35)$ e comportamento sedentário superior a duas horas semanais $(\mathrm{RP}=0,82)$ apresentaram associação significativa com o atendimento ao critério de saúde para aptidão cardiorrespiratória.

Os resultados referente aos escolares com idade igual ou superior a 14 anos são apresentados na Tabela 3. Após serem ajustadas para todas as variáveis do modelo, as variáveis sexo feminino $(\mathrm{RP}=0,35)$, sobrepeso $(R P=0,25)$ e prática esportiva algumas vezes $(R P=1,96)$ e frequentemente/sempre $(\mathrm{RP}=2,09)$ se mantiveram associadas ao atendimento do critério de saúde para aptidão cardiorrespiratória.

As variáveis condição socioeconômica ( $P=0,924$ e $P=0,989$ ), transporte ativo ( $P=0,671$ e 0,931$)$ e comportamento sedentário no fim de semana ( $P=0,368$ e 0,301), para escolares com idade inferior e igual ou superior a 14 anos respectivamente, não foram descritas nas Tabelas 2 e 3 por não apresentarem associação significativa na análise bivariada em ambas as faixas etárias.

\section{DISCUSSÃO}

Os principais resultados desse estudo foram que em escolares com idade inferior a 14 anos as variáveis sexo, sobrepeso, percepção de atividade física, atividade física moderada a vigorosa semanal, participação nas aulas de Educação Física e comportamento sedentário apresentaram associação significativa com a aptidão cardiorrespiratória. Para os escolares com idade igual ou superior a 14 anos, as variáveis significativamente associadas com o atendimento ao critério para aptidão cardiorrespiratória foram o sexo, sobrepeso e a prática esportiva. 
Tabela 1. Características da amostra de acordo com a idade.

\begin{tabular}{|c|c|c|}
\hline \multirow[b]{2}{*}{ Variáveis } & \multicolumn{2}{|c|}{ Idade } \\
\hline & $\begin{array}{c}<14 \text { anos } \\
n=358\end{array}$ & $\begin{array}{c}\geq 14 \text { anos } \\
n=378\end{array}$ \\
\hline \multicolumn{3}{|l|}{ Sexo } \\
\hline Masculino & 50,6 & 49,2 \\
\hline Feminino & 49,4 & 50,8 \\
\hline \multicolumn{3}{|l|}{ Condição socioeconômica } \\
\hline Alta & 14,9 & 11,7 \\
\hline Média & 54,9 & 52,9 \\
\hline Baixa & 30,2 & 35,4 \\
\hline \multicolumn{3}{|l|}{ Escolaridade paterna } \\
\hline Fundamental completo & 12,3 & 19,0 \\
\hline Ensino médio completo & 58,1 & 67,7 \\
\hline Superior completo & 18,7 & 13,2 \\
\hline \multicolumn{3}{|l|}{ Número de irmãos } \\
\hline 0 & 12,3 & 9,3 \\
\hline $1-2$ & 61,4 & 65,1 \\
\hline$\geq 3$ & 26,3 & 25,6 \\
\hline \multicolumn{3}{|l|}{ Estado nutricional } \\
\hline Sobrepeso & 22,9 & 21,2 \\
\hline Eutrófico & 77,1 & 78,8 \\
\hline \multicolumn{3}{|l|}{ Percepção de atividade física ${ }^{*}$} \\
\hline Baixa/muito baixa & 23,7 & 37,8 \\
\hline lgual & 41,6 & 33,9 \\
\hline Elevada/muito elevada & 34,6 & 28,3 \\
\hline \multicolumn{3}{|l|}{ Atividade física semanal } \\
\hline $0-2 h$ & 52,5 & 60,6 \\
\hline $3-6 h$ & 35,5 & 25,4 \\
\hline$\geq 7 \mathrm{~h}$ & 12,0 & 14,0 \\
\hline \multicolumn{3}{|l|}{$\begin{array}{c}\text { Participação em aulas } \\
\text { de Educação Física }\end{array}$} \\
\hline Não & 11,7 & 18,5 \\
\hline Sim & 88,3 & 81,5 \\
\hline \multicolumn{3}{|c|}{ Local para prática de atividade física } \\
\hline Não possui & 18,8 & 21,4 \\
\hline Possui apenas 1 & 37,4 & 40,2 \\
\hline Possui mais que 1 & 42,7 & 38,4 \\
\hline \multicolumn{3}{|l|}{ Prática esportiva } \\
\hline Nunca/raramente & 24,9 & 39,2 \\
\hline Algumas vezes & 33,2 & 33,3 \\
\hline Frequentemente/sempre & 41,9 & 27,5 \\
\hline \multicolumn{3}{|l|}{ Transporte ativo } \\
\hline$<30 \mathrm{~min} / \mathrm{dia}$ & 67,3 & 73,4 \\
\hline$\geq 30 \mathrm{~min} / \mathrm{dia}$ & 30,7 & 26,6 \\
\hline \multicolumn{3}{|l|}{ Comportamento sedentário $^{\dagger}$} \\
\hline$<2 h$ & 25,4 & 18,0 \\
\hline$\geq 2 h$ & 74,6 & 82,0 \\
\hline
\end{tabular}

* Comparado à pessoas de mesma idade; ${ }^{\dagger}$ durante a semana.

Já foi descrito, em outro estudo utilizando método, instrumentos e pontos de corte semelhantes ao do presente, que a proporção do atendimento do critério para aptidão cardiorrespiratória é menor em escolares do sexo feminino ${ }^{11}$ e com o aumento dos anos a proporção de atendimento do critério também diminui ${ }^{5,6}$. Provavelmente a atividade física é um fator que se associa a essas alterações de acordo com o sexo e com a idade, haja vista que, como demonstrado em um estudo realizado com adolescentes da cidade de São Paulo ${ }^{20}$, há maior prevalência de inatividade física em escolares do sexo feminino e com maior idade. Outro aspecto relevante a ser apontado é que em ambos os sexos o comportamento sedentário aumenta com a idade, como demonstrado em um estudo longitudinal utilizando acelerometria em
Tabela 2. Razão de prevalência (RP) bruta e ajustada e os intervalos de confiança de 95\% (IC95\%) dos fatores associados ao atendimento do critério para aptidão cardiorrespiratória nos escolares com idade inferior a 14 anos.

\begin{tabular}{|c|c|c|c|c|}
\hline \multirow[t]{2}{*}{ Variáveis } & \multirow[t]{2}{*}{ Atende \% } & \multirow[t]{2}{*}{$\mathbf{P}$} & \multicolumn{2}{|c|}{ RP } \\
\hline & & & $\begin{array}{c}\text { Bruta } \\
\text { RP (IC 95\%) }\end{array}$ & $\begin{array}{c}\text { Ajustada } \\
\text { RP (IC 95\%) }\end{array}$ \\
\hline \multicolumn{5}{|l|}{ Sexo } \\
\hline Masculino & 82,9 & $<0,001$ & 1,00 & 1.00 \\
\hline Feminino & 57,6 & & $0,69(0,60-0,80)$ & $0,79(0,68-0,91)$ \\
\hline \multicolumn{5}{|l|}{ Escolaridade paterna } \\
\hline Fundamental completo & 63,9 & 0,044 & 1,00 & 1.00 \\
\hline Ensino médio completo & 70,2 & & $1,09(0,91-1,31)$ & $1,07(0,91-1,27)$ \\
\hline Superior completo & 79,1 & & $1,24(1,01-1,51)$ & $1,18(0,98-1,40)$ \\
\hline \multicolumn{5}{|l|}{ Número de irmãos } \\
\hline 0 & 77,3 & 0,349 & & - \\
\hline $1-2$ & 65,3 & & & - \\
\hline$\geq 3$ & 78,7 & & & - \\
\hline \multicolumn{5}{|l|}{ Estado nutricional } \\
\hline Sobrepeso & 56,1 & 0,001 & $0,75(0,61-0,92)$ & $0,73(0,61-0,89)$ \\
\hline Eutrófico & 74,6 & & 1,00 & 1.00 \\
\hline \multicolumn{5}{|l|}{$\begin{array}{l}\text { Percepção de } \\
\text { atividade física* }\end{array}$} \\
\hline Baixa/muito baixa & 54,1 & 0,002 & 1,00 & 1.00 \\
\hline lgual & 75,2 & & $1,38(1,11-1,72)$ & $1,26(1,03-1,53)$ \\
\hline Elevada/muito elevada & 75,8 & & $1,40(1,12-1,74)$ & $1,21 \quad(0,98-1,48)$ \\
\hline \multicolumn{5}{|l|}{ Atividade física semanal } \\
\hline $0-2 \mathrm{~h}$ & 60,6 & $<0,001$ & 1,00 & 1.00 \\
\hline $3-6 h$ & 81,9 & & $1,35(1,17-1,55)$ & $1,21(1,05-1,38)$ \\
\hline$\geq 7 \mathrm{~h}$ & 79,1 & & $1,30(1,07-1,58)$ & $1,10(0,91-1,33)$ \\
\hline \multicolumn{5}{|l|}{$\begin{array}{c}\text { Participação em aulas } \\
\text { de Educação Física }\end{array}$} \\
\hline Não & 52,4 & 0,007 & 1,00 & 1.00 \\
\hline Sim & 72,8 & & $1,38(1,03-1,86)$ & $1,35(1,03-1,76)$ \\
\hline \multicolumn{5}{|l|}{$\begin{array}{l}\text { Local para prática de } \\
\text { atividade física }\end{array}$} \\
\hline Não & 64,8 & 0,029 & 1,00 & 1,00 \\
\hline Apenas 1 & 65,7 & & $1,01(0,82-1,25)$ & $1,04(0,86-1,25)$ \\
\hline Mais que 1 & 77,1 & & $1,19(0,98-1,44)$ & $1,14(0,96-1,36)$ \\
\hline \multicolumn{5}{|l|}{ Prática esportiva } \\
\hline Nunca/raramente & 57,3 & $<0,001$ & 1,00 & 1,00 \\
\hline Algumas vezes & 67,2 & & $1,17(0,94-1,46)$ & $1,09(0,89-1,33)$ \\
\hline Frequentemente/sempre & 80,7 & & $1,40(1,15-1,71)$ & $1,12(0,94-1,35)$ \\
\hline \multicolumn{5}{|l|}{$\begin{array}{c}\text { Comportamento } \\
\text { sedentário }^{\dagger}\end{array}$} \\
\hline$<2 \mathrm{~h}$ & 82,4 & 0,004 & 1,00 & 1,00 \\
\hline$\geq 2 h$ & 66,3 & & $0,80(0,70-0,91)$ & $0,82(0,73-0,94)$ \\
\hline
\end{tabular}

* Comparado à pessoas de mesma idade; ${ }^{\dagger}$ durante a semana; P referente ao teste de Qui-Quadrado.

adolescentes do Reino Unido ${ }^{21}$, o que provavelmente também pode contribuir com o menor atendimento do critério para aptidão cardiorrespiratória apresentada pelos escolares mais velhos no presente estudo $(70,4$ vs 19,6).

Uma revisão sistemática descreveu que apesar do treinamento físico aumentar a aptidão cardiorrespiratória, a relação com a atividade física habitual ainda não é consenso na literatura ${ }^{22}$. No entanto, estudos recentes realizados nas cidades de Florianópolis-SC ${ }^{8}$, Montes Claros-MG ${ }^{11}$ e Curitiba-PR ${ }^{10}$, demonstraram associação positiva entre ser fisicamente ativo com a aptidão cardiorrespiratória em crianças e adolescentes. No presente estudo, a associação entre atividade física e aptidão cardiorrespiratória foi encontrada apenas para os escolares 
Tabela 3. Razão de prevalência (RP) bruta e ajustada e os intervalos de confiança de 95\% (IC95\%) dos fatores associados ao atendimento do critério para aptidão cardiorrespiratória nos escolares com idade superior a 14 anos.

\begin{tabular}{|c|c|c|c|c|}
\hline \multirow[t]{2}{*}{ Variáveis } & \multirow[t]{2}{*}{ Atende $\%$} & \multirow[t]{2}{*}{$\mathbf{P}$} & \multicolumn{2}{|c|}{ RP } \\
\hline & & & $\begin{array}{c}\text { Bruta } \\
\text { RP (IC 95\%) }\end{array}$ & $\begin{array}{c}\text { Ajustada } \\
\text { RP (IC 95\%) }\end{array}$ \\
\hline \multicolumn{5}{|l|}{ Sexo } \\
\hline Masculino & 31,7 & $<0,001$ & 1,00 & 1,00 \\
\hline Feminino & 7,8 & & $0,24(0,14-0,41)$ & $0,35(0,19-0,63)$ \\
\hline \multicolumn{5}{|l|}{ Escolaridade paterna } \\
\hline Fundamental incompleto & 18,1 & 0,597 & - & - \\
\hline Ensino médio completo & 19,5 & & - & - \\
\hline Superior completo & 22,0 & & - & - \\
\hline \multicolumn{5}{|l|}{ Número de irmãos } \\
\hline 0 & 14,3 & 0,002 & 1,00 & 1,00 \\
\hline $1-2$ & 14,3 & & $1,08(0,45-2,56)$ & $1,06(0,49-2,28)$ \\
\hline$\geq 3$ & 32,0 & & $2,23(0,94-5,30)$ & $2,00(0,93-4,29)$ \\
\hline \multicolumn{5}{|l|}{ Estado nutricional } \\
\hline Sobrepeso & 5,0 & $<0,001$ & $0,21(0,08-0,56)$ & $0,25(0,09-0,66)$ \\
\hline Eutrófico & 23,5 & & 1,00 & 1,00 \\
\hline \multicolumn{5}{|l|}{$\begin{array}{l}\text { Percepção de } \\
\text { atividade física }^{*}\end{array}$} \\
\hline Baixa/muito baixa & 11,9 & $<0,001$ & 1,00 & 1,00 \\
\hline lgual & 18,8 & & $1,57(0,88-2,80)$ & $0,86(0,49-1,49)$ \\
\hline Elevada/muito elevada & 30,8 & & $2,59(1,52-4,40)$ & $1,05(0,61-1,81)$ \\
\hline \multicolumn{5}{|l|}{ Atividade física semanal } \\
\hline $0-2 \mathrm{~h}$ & 10,5 & $<0,001$ & 1,00 & 1,00 \\
\hline $3-6 h$ & 36,5 & & $3,47(2,19-5,52)$ & $1,48(0,86-2,52)$ \\
\hline$\geq 7 \mathrm{~h}$ & 28,3 & & $2,70(1,52-4,78)$ & $1,03(055-1,93)$ \\
\hline \multicolumn{5}{|l|}{$\begin{array}{c}\text { Participação em aulas } \\
\text { de Educação Física }\end{array}$} \\
\hline Não & 12,9 & 0,116 & 1,00 & 1,00 \\
\hline Sim & 21,2 & & $1,64(0,85-3,13)$ & $1,30(0,74-2,28)$ \\
\hline \multicolumn{5}{|l|}{$\begin{array}{l}\text { Local para prática de } \\
\text { atividade física }\end{array}$} \\
\hline Não possui & 16,0 & 0,348 & - & - \\
\hline Possui apenas 1 & 19,7 & & - & - \\
\hline Possui mais que 1 & 21,4 & & - & - \\
\hline \multicolumn{5}{|l|}{ Prática esportiva } \\
\hline Nunca/raramente & 7,4 & $<0,001$ & 1,00 & 1,00 \\
\hline Algumas vezes & 20,6 & & $2,77(1,42-5,39)$ & $1,96(1,00-3,81)$ \\
\hline Frequentemente/sempre & 35,6 & & $4,78(2,56-8.94)$ & $2,09(1,01-4,31)$ \\
\hline \multicolumn{5}{|l|}{$\begin{array}{c}\text { Comportamento } \\
\text { sedentário }^{\dagger}\end{array}$} \\
\hline$<2 \mathrm{~h}$ & 19,1 & 0,916 & - & - \\
\hline$\geq 2 \mathrm{~h}$ & 19,7 & & - & - \\
\hline
\end{tabular}

* Comparado à pessoas de mesma idade; ${ }^{\dagger}$ durante a semana; P referente ao teste de Qui-Quadrado.

com idade inferior a 14 anos. Apesar de não ter sido investigado no presente estudo, adolescentes mais velhos se engajam em práticas corporais as quais não têm como objetivo aumentar a aptidão cardiorrespiratória, tais como treinamento de força ${ }^{23}$, fato que pode explicar a não associação encontrada. Isso se torna relevante pelo fato de que os tipos de atividades físicas realizadas não foram discriminadas pelo instrumento utilizado.

No presente estudo, ao contrário da atividade física, a prática esportiva apresentou associação significativa com a aptidão cardiorrespiratória dos escolares com idade igual ou superior a 14 anos. No Brasil, os esportes mais difundidos entre os escolares são os coletivos, futebol, futsal, basquetebol, handebol e voleibol. Com exceção do voleibol, são esportes que demandam intensidade moderada a vigorosa e são de longa duração, fato que beneficia a saúde de adolescentes como, por exemplo, resultando em aumento da aptidão cardiorrespiratória ${ }^{24}$. As informações disponíveis da literatura, somados com os encontrados no presente estudo, indicam a necessidade da prática de atividade física moderada à vigorosa e da prática esportiva com objetivo de prevenir os agravos à saúde relacionados à baixa aptidão cardiorrespiratória',2.

Este foi o primeiro estudo a demonstrar associação positiva entre participação nas aulas de Educação Física com a aptidão cardiorrespiratória em escolares brasileiros. Já havia sido descrito associação positiva entre participação de 200 min em aulas de Educação física a cada 10 dias com o atendimento do critério para aptidão cardiorrespiratória em escolares americanos ${ }^{25}$. Em jovens da cidade de Curitiba-PR foram encontradas associações entre atividade física superior a 30 min por aula nos meninos com a aptidão cardiorrespiratória ${ }^{10}$. Em parte os resultados do presente estudo corroboram com os prévios ${ }^{10}$, uma vez que provavelmente os escolares que participam das aulas realizam maiores quantidades de atividade física comparados aos não participantes. No Brasil comumente são realizadas duas aulas semanais com duração de 50 min cada, e provavelmente, a realização do exercício físico de forma orientada pode ser responsável pela maior proporção do atendimento do critério para aptidão cardiorrespiratória em escolares que participam das aulas de Educação Física.

Escolares praticantes de atividade física, de esportes e que participam das aulas de Educação Física provavelmente possuem adaptações similares aos jovens submetidos ao treinamento aeróbio, como por exemplo, maior pico de consumo de oxigênio, maior volume de ejeção ventricular, cinética de consumo de oxigênio mais rápida nas alterações de intensidade do exercício, menor acúmulo de lactato em exercício submáximo e maior função oxidativa dos músculos treinados ${ }^{26}$. Tais adaptações ao treinamento favorecem o desempenho da tarefa imposta pelo teste utilizado (PACER), que é progressivo até a exaustão.

O sobrepeso apresentou associação negativa com a aptidão cardiorrespiratória independente da faixa etária. Jovens com sobrepeso possuem limitação para aumentar a demanda metabólica requerida para movimentar a maior massa corporal que possuem. Consequentemente, apresentam valores semelhantes de consumo de oxigênio, no limiar de lactato e no esforço máximo comparado a adolescentes eutrófico ${ }^{27}$. Este fato torna-se relevante, uma vez que o teste PACER utilizado no presente estudo é progressivo e máximo. Além disso, o mesmo estudo ${ }^{27}$ demonstrou que adolescentes com sobrepeso consomem maior quantidade relativa de oxigênio durante o exercício submáximo devido à maior massa corporal a ser movimentada, o que pode diminuir o tempo necessário para alcançar a exaustão. Além das características já citadas, jovens com sobrepeso também apresentam menor resposta da cinética do consumo de oxigênio em exercício moderado²8.

Outro fator que apresentou associação com o atendimento do critério para aptidão cardiorrespiratória foi a percepção de atividade física igual às pessoas de mesma idade. A literatura apontou que aproximadamente $40 \%$ dos escolares britânicos superestimam sua atividade física habitual2 ${ }^{29}$. Provavelmente, por esse motivo, no presente estudo apenas os escolares que apresentaram percepção igual às pessoas de mesma idade apresentaram razão de prevalência superior quando comparada aos que apresentam baixa percepção de atividade física.

No presente estudo, o comportamento sedentário se associou inversamente com o atendimento ao critério de saúde para aptidão cardiorrespiratória nos escolares mais jovens. A associação entre comportamento sedentário e aptidão cardiorrespiratória já foi descrita por estudos recentes e a associação parece ser independente da atividade física ${ }^{8,10}$. Dessa forma, além de incentivar a prática de atividade física há 
necessidade de reduzir o comportamento sedentário há no máximo duas horas diárias.

Dentre as limitações do presente estudo destacam-se a utilização de questionário e teste indireto para estimativa da aptidão cardiorrespiratória. Ainda, não foram analisados os tipos de atividades físicas, conteúdos das aulas de Educação Física e os esportes que os escolares praticavam, fato que pode limitar a generalização dos resultados encontrados.

\section{CONCLUSÃO}

Pode-se concluir que para os escolares com idade inferior a 14 anos, o sexo feminino, sobrepeso, percepção de atividade física per- cepção de atividade física igual às pessoas de mesma idade, atividade física moderada a vigorosa de 3 a 6 horas semanais, participação nas aulas de Educação Física e comportamento sedentários superior a duas horas semanais são fatores associados à aptidão cardiorrespiratória. Para os adolescentes mais velhos os fatores que apresentaram associação com a aptidão cardiorrespiratória foram o sexo, sobrepeso e prática esportiva.

Todos os autores declararam não haver qualquer potencial conflito de interesses referente a este artigo.

CONTRIBUIÇÕES DOS AUTORES: Cada autor contribuiu individual e significantemente para o desenvolvimento deste artigo. DHCC (0000-0001-6211-7069)* e PFF (0000-0002-3486-6473)* atuaram na coleta, análise e interpretação dos dados, elaboração, revisão crítica e final do manuscrito. JWS (0000-0002-5507-6809)* e ARO (0000-0003-4503-9083)* colaboraram na interpretação e discussão dos resultados, revisão crítica e aprovação final do manuscrito. *Número ORCID (Open Researcher and Contributor ID).

\section{REFERÊNCIAS}

1. Ruiz JR, Huybrechts I, Cuenca-García M, Artero EG, Labayen I, Meirhaeghe A, et al. Cardiorespiratory fitness and ideal cardiovascular health in European adolescents. Heart. 2015;101(10):766-73.

2. Rieck T, Jackson A, Martin SB, Petrie T, Greenleaf C. Health-related fitness, body mass index, and risk of depression among adolescents. Med Sci Sports Exerc. 2013;45(6):1083-8,

3. Hurtig-Wennlöf A, Ruiz JR, Harro M, Sjöström M. Cardiorespiratory fitness relates more strongly than physical activity to cardiovascular disease risk factors in healthy children and adolescents: the European Youth Heart Study. Eur J Cardiovasc Prev Rehabil. 2007:14(4):575-81.

4. Rizzo NS, Ruiz JR, Hurtig-Wennlöf A, Ortega FB, Sjöström M. Relationship of physical activity, fitness, and fatness with clustered metabolic risk in children and adolescents: the European youth heart study. J Pediatr. 2007;150(4):388-94.

5. Guedes DP, Miranda Neto JT, Germano JM, Lopes VM, Silva AJR. Aptidão física relacionada à saúde de escolares: programa Fitnessgram. Rev Bras Med Esporte 2012;18(2):72-6.

6. Petroski EL, Silva AF, Silva JMFL, Pelegrini A. Health-related physical fitness and associated sociodemographic factors in adolescents from a Brazilian state capital. Human Movement. 2012;13(2):139-46

7. Petroski EL, Silva AF, Rodrigues AB, Pelegrini A. Associação entre baixos níveis de aptidão física e fatores sociodemográficos em adolescentes de área urbanas e rurais. Motricidade 2012;8(1):5-13.

8. Silva DAS, Tremblay MS, Pelegrini A, Silva JMFL, Petroski EL. Low aerobic fitness in Brazilian adolescents. Rev Bras Med Esporte. 2015;21(2):94-8.

9. Minatto G, Silva DAS, Pelegrini A, Fidelix YL, Silva AF, Petroski EL. Aptidão cardiorrespiratória, indicadores sociodemográficos e estado nutricional em adolescentes. Rev Bras Med Esporte. 2015;21(1):12-6.

10. Barbosa Filho VC, Lopes Ada S, Bozza R, Rech CR, de Campos W. Correlates of cardiorespiratory and muscular fitness among Brazilian adolescents. Am J Health Behav. 2014;38(1):42-52.

11. Guedes DP, Miranda Neto J, Lopes VP, Silva AJ. Health-related physical fitness is associated with selected sociodemographic and behavioral factors in Brazilian school children. J Phys Act Health. 2012;9(4):473-80

12. Straatmann S, Veiga VGV. Cardiorespiratory Fitness, Physical Activity, and Indicators of Adiposity in Brazilian Adolescents. Human Movement. 2015;16(2):64-70.

13. Baecke JA, Burema J, Frijters JE. A short questionnaire for the measurement of habitual physical activity in epidemiological studies. Am J Clin Nutr. 1982;36(5):936-42.

14. Associação Brasileira de Empresas de Pesquisa (ABEP). Critério de Classificação Econômica do Brasil, 2012. Disponível em: http://www.abep.org. Acesso em 12 de dez/2012.
15. Coledam DH, Ferraiol PF, Pires Junior $R$, dos-Santos JW, Oliveira AR. Factors associated with participation in sports and physical education among students from Londrina, Paraná State, Brazil. Cad Saude Publica. 2014;30(3):533-45.

16. Léger LA, Mercier D, Gadoury C, Lambert J. The multistage 20 metre shuttle run test for aerobic fitness. J Sports Sci. 1988;6(2):93-101

17. Meredith MD, Welk GJ. Fitnessgram/Activitygram test administration manual. 4a. ed. Dallas, TX: The Cooper Institute; 2010.

18. World Health Organization (WHO). Physical status: the use and interpretation of anthropometry. Report of a WHO Expert Committee - Technical Report Series n 854. Geneva: WHO; 1995.

19. Cole TJ, Lobstein T. Extended international (IOTF) body mass index cut-offs for thinness, overweight and obesity. Pediatr Obes. 2012;7(4):284-94.

20. Ceschini FL, Andrade DR, Oliveira LC, Araújo Júnior JF, Matsudo VK. Prevalence of physical inactivity and associated factors among high school students from state's public schools. J Pediatr (Rio Janeiro). 2009;85(4):301-6

21. Mitchell JA, Pate RR, Dowda M, Mattocks C, Riddoch C, Ness AR, Blair SN. A prospective study of sedentary behavior in a large cohort of youth. Med Sci Sports Exerc. 2012;44(6):1081-7.

22. Armstrong $\mathrm{N}$, Tomkinson $\mathrm{G}$, Ekelund U. Aerobic fitness and its relationship to sport, exercise training and habitual physical activity during youth. Br J SportsMed. 2011;45(11):849-58.

23. Santos AP, Marinho DA, Costa AM, Izquierdo M, Marques MC. The effects of concurrent resistance and endurance training follow a detraining period in elementary school students. J Strength Cond Res. 2012;26(6):1708-16.

24. Ara I, Moreno LA, Leiva MT, Gutin B, Casajús JA. Adiposity, physical activity, and physical fitness among children from Aragón, Spain. Obesity (Silver Spring). 2007;15(8):1918-24

25. Sanchez-Vaznaugh EV, Sánchez BN, Rosas LG, Baek J, Egerter S. Physical education policy compliance and children's physical fitness. Am J Prev Med. 2012;42(5):452-9.

26. Armstrong N, Barker AR. Endurance training and elite young athletes. Med Sport Sci. 2011;56:59-83.

27. Norman AC, Drinkard B, McDuffie JR, Ghorbani S, Yanoff LB, Yanovski JA. Influence of excess adiposity on exercise fitness and performance in overweight children and adolescents. Pediatrics. 2005;115(6):e690-6.

28. Potter CR, Zakrzewski JK, Draper SB, Unnithan VB. The oxygen uptake kinetic response to moderate intensity exercise in overweight and non-overweight children. Int J Obes (Lond). 2013;37(1):101-6.

29. Corder K, van Sluijs EM, McMinn AM, Ekelund U, Cassidy A, Griffin SJ. Perception versus reality awareness of physical activity levels of British children. Am J Prev Med. 2010;38(1):1-8, 